\title{
GIS-BIM Framework for Integrating Urban Systems, Waste Stream and Algal Cultivation in Residential Construction
}

\author{
Daniel Castro-Lacouture ${ }^{a}$, Steven Jige Quan ${ }^{b}$, and Perry Pei-Ju Yang ${ }^{c}$ \\ ${ }^{a}$ Associate Professor, School of Building Construction, Georgia Institute of Technology, Atlanta, GA 30332 USA \\ ${ }^{b}$ Graduate Research Assistant, Built Environment Sustainable Technology (BEST) Laboratory, Georgia Institute of \\ Technology, Atlanta, GA 30332 USA \\ ${ }^{c}$ Associate Professor, School of Architecture, School of City and Regional Planning, Georgia Institute of \\ Technology, Atlanta, GA 30332 USA \\ E-mail: dcastro@gatech.edu, steven@gatech.edu,perry.yang@coa.gatech.edu
}

\begin{abstract}
Among alternative energy sources for residential buildings, algae technology has emerged as a promising option due to its closed-loop configuration and the ability to produce biofuel energy while reducing waste stream flow and capturing carbon. Furthermore, this technology has the potential of integrating resource and waste management, and can be complemented with other alternatives, such as photovoltaic, wind or fuel cells. This paper provides a framework for integrating information from geographic information systems, building information models, construction schedules, construction cost estimates, and constructability reviews. The integration is aimed at designing an algae-powered residential building environment at the level of urban neighbourhood, in which the algae technology is taken as a design intervention to promote energy performance and carbon reduction within the urban system. This framework couples the design intervention with impact simulations influenced by geographic contexts, construction considerations, and digital building technology. By extending the system boundary from a closed algae cultivation system to an open neighbourhood-scale urban environment, urban renewable resources such as energy, water, material and carbon flows are connected to the algae cultivation process. The framework would further advance the possibilities for sharing information among planners, architects, engineers and construction managers for innovative closed-loop sustainable energy systems in residential construction. This approach will address challenges such as cost, governmental incentives, regulatory barriers, or need of research and development that could overcome limitations for automating predesign, design, construction and facility management processes.
\end{abstract}

Keywords -

Algae technology, GIS, BIM, residential construction

\section{Introduction}

There is a potential for the use of algal cultivation and processing to manage the liquid and solid waste from a residential community and recycle the carbon stream into energy of sufficient quality to power the dwellings in a sustainable manner $[1,2]$. Once maximum reductions are made with respect to resource utilization and maximum efficiencies are realized using green, conservation-driven design principles, the evaluation of the waste streams must be considered for opportunities for reuse, recycling, and treatment processes that maximize the extraction of resources. Novel sources of inputs may then be developed that take advantage of the holistic approach to resource management.

Primary residential waste streams include wastewater and municipal solid waste. In addition, thermal and other energy losses due to inefficiencies require additional demands on resource inputs. In addition to the current first-choice renewable energy generating technologies available to residential communities (e.g., solar photovoltaic, solar thermal, wind turbine), novel technologies are currently under development that may be able to address the desire for sustainable energy generation while, at the same time, address waste stream management issues through resource recovery. Figure 1 identifies selected materials and energy inputs and outputs for a typical single-family home. In addition to the selection of an appropriate strain of microalgae, algae cultivation requires four primary inputs: water, nutrients (primarily nitrogen and phosphorous), carbon (primarily carbon dioxide), and sunlight. 


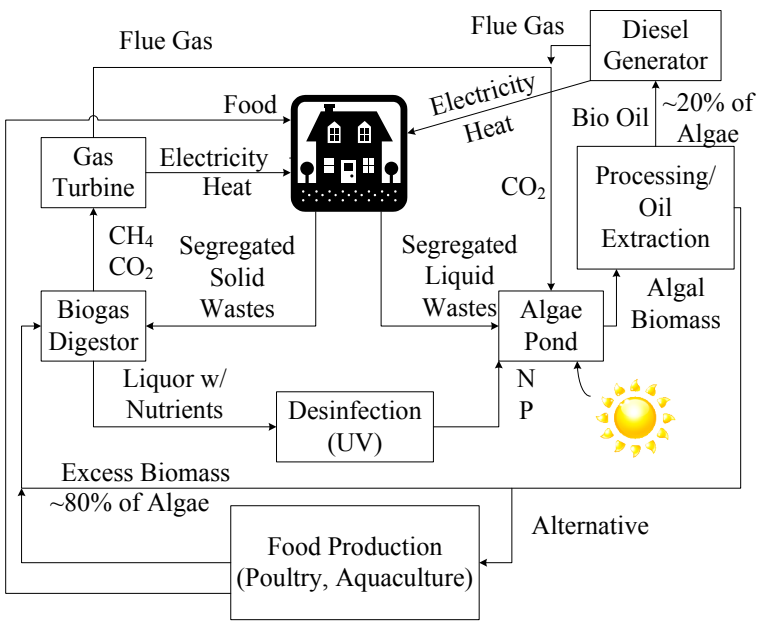

Figure 1. Material and energy flows in algae housing

Solid wastes that were readily biodegradable are directed to the biogas digester where anaerobic bacteria converts the majority of the organic carbon into methane and carbon dioxide. These gases are combusted in a gas turbine where electricity and heat are supplied to the residence. The flue gases (now $\mathrm{CO} 2$ and residual N2) are be sparged into the algae cultivation pond. The liquid waste from the digester (liquor) is very rich in organic nutrients and is disinfected using UV technology and the residual nutrients added to the algae pond. Harvested algae is processed to remove natural lipids, assumed to be $20 \%$ of the algae mass. That oil can be used directly in a diesel generator, again to provide electricity and heat to the residence, and the flue gas from the diesel generator is sparged into the algae pond. The remaining $80 \%$ of the algal biomass is then directed to the biogas digester, providing additional methane for the gas turbine. The $80 \%$ fraction of algal biomass may also be used directly as food or animal feed. Examples of feed options include small food-producing animals, like chickens or ducks, or aquaculture opportunities, such as shrimp or fish farming. The wastes from the food production would also be amenable to inclusion in the biogas digester to provide additional biomass for energy generation and nutrient recycling. Perspectives for the feasibility of microalgae technology has been discussed elsewhwere [1, 2, 3]. However, a more integrative system of sustainable housing and development presumes the interrelationship of parameters and metrics between planning, architecture and building construction.

\section{Integrating waste stream, hydrological cycle and solar energy through GIS}

The design of the algae-powered system should be applicable to actual contexts in urban, suburban and rural settings. Actual sites are selected along the urban gradient, with the measure of population density, land use, land cover, and other spatial information that can be managed in Geographic Information System (GIS). A set of planning parameters and assessment tools are used to evaluate the environmental performance of the neighbourhood-scale operation that focuses on waste stream, hydrological recycle and solar availability assessment to understand how the algae-powered house can be adapted to different settings along the urban gradient, as seen in Table 1 . Waste stream $=$ population $x$ 40-60 gal/day. Annual surface runoff and infiltration rate is estimated based on local coefficients. Waste stream, hydrological cycle and solar availability associated with the urban gradient are the three selected planning parameters for evaluating potential impact on the operation, performance and feasibility of the algaepowered setting. As the neighbourhood scale, density and typology change, the processes of the inputs and outputs of flows of waste, water and solar energy would change accordingly.

Table 1. Urban, suburban and rural neighbourhood typologies and planning parameters

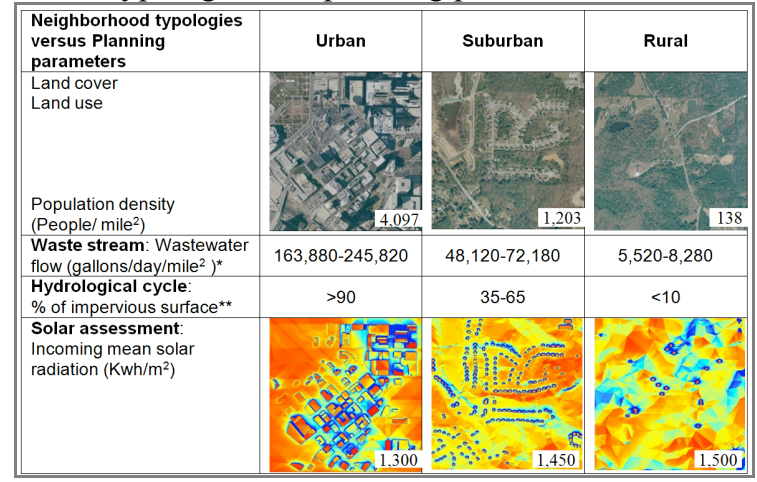

\subsection{Waste stream}

The selection of neighbourhood scale, population density and land use will determine the amount of biomass produced and the energy consumption pattern, and will affect the design, operation, performance and feasibility of the Algae-powered system. For example, In the region of Atlanta, Georgia, United States, the daily wastewater flow of an urban neighbourhood is about three times that of a suburban neighbourhood, and can go over thirty times that of a rural area based on the same size territory as shown in Figure 3. The relationship between neighbourhood scale, population 
density and waste stream management will need to be established.

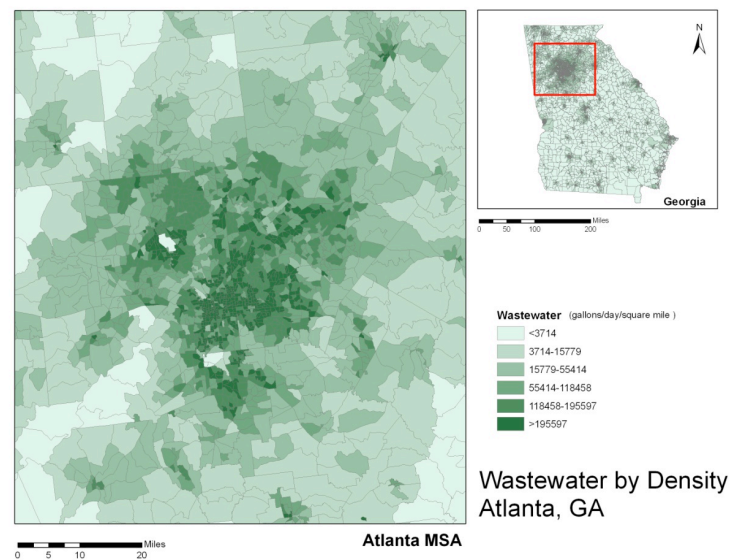

Figure 3. Intensity of Waste Water Flow in Atlanta, Georgia, United States

\subsection{Hydrological cycle}

Hydrological cycle: the hydrological balance and the sewage flows will affect the amount of water required to manage algae ponds. To prevent the algae ponds from drying out or becoming too salty, conditions that would kill the algae, a steady supply of freshwater is needed to replenish the evaporating water [4]. The hydrological conditions of detention, ground water discharge and the grey water reuse will be explored. The urban site tends to have higher level of impervious surfaces and lower level of infiltration, and thus contains less groundwater availability to contribute to the base flow between storms especially in the dry period [5]. However, urban sites normally contain higher wastewater flow that provides potential steady supply of grey water for re-use in the algae pond with on-site waste water treatment such as constructed wetlands. In suburban and rural settings, the hydrological cycle is to be designed and managed by low impact development that works with nature to manage storm water as close to its source as possible [6]. The design of algae pond tends to choose a landscape approach that is to be integrated in the landscape ecological network. The research also adopts land cover based performance measure to connect relationship between the land cover type information and the annual hydrological parameters such as annual mean surface runoff and infiltration [7]. The hydrological model helps to determine location of algae pond and to quantify the area required for production of algae.

\subsection{Solar availability}

Incoming solar radiation or insolation received from the sun is the primary energy source that drives algae biological processes. The algae need sunlight to photosynthesize. Topography and built environment are major factors that determine the spatial variability of insolation. Across the urban gradient from urban, suburban to rural, the amount of insolation decreases accordingly, as seen in Table 1. In urban settings, the amount of insolation is affected by not only topography but also by surrounding buildings. While in the rural setting, the main factor determining insolation is orientation, slope, aspect and elevation. The solar availability analysis will also provide the information to support the location decision of the algae pond.

\subsection{BIM-GIS integration}

The integration of algal cultivation system and urban system calls for a new information technology management system to facilitate the construction process. Such information management system is spatial and locational, and moreover it deals with issues across different spatial scales. Such information system should involve the technologies that could manage the system data with the urban spatial modelling.

However, there is still a big gap between the spatial information technologies that apply at the micro spatial scale and at the macro scale. At the building level as the micro scale in the urban system, BIM (Building Information Modelling) goes beyond the traditional CAD (Computer Aided Drafting) approach for 2D representation to a new paradigm for placing real information in the model database to virtually construct a building [8]. BIM is designed to support the building maintenance and the life-cycle development [9]. At the urban level as the macro scale, GIS (Geographic Information System) aims at managing the database with spatial reference [10]. Different purposes and emphasis on different scales of these two information technologies lead to their various capacities in dealing with information at different levels and their distinct methods in locating objects. First, BIM provides strong functions to handle the "indoor" data, at a relatively micro spatial scale, while GIS offers many tools to organize the "outdoor" data, e.g. streets, land parcels and terrains, at the macro spatial scale. Second, BIM places the building as the system boundary and models the relative coordinates of the objects within the system, while GIS assumes the Earth as the system and places every object with the geographic coordinate systems and the world map projection. Third, BIM emphasizes the life cycle of the building construction and therefore has a clear temporal dimension, while GIS focuses more 
on managing the interaction of spatial, social and economic information at a given time. Fourth, and the most important, ArcGIS has the advantage of network analysis based on geo-locations, while BIM is relatively weak on this aspect. Besides, their data formats are based on different protocols and therefore cannot be directly opened by each other.

Due to these differences of the two informational technologies, they are usually utilized at separate academic or practice fields. ArcGIS is often used at urban planning, transportation planning and urban design analysis, while BIM is commonly applied to architectural design and building construction management, though it could be related to planning parameters at larger scales [8].

However, in the combined algae-urban system, the information system faces new challenges to deal with cross-scale and system-oriented issues, with the following questions at hand: where is the resource? How are algal cultivation systems of different levels integrated to buildings and neighbourhood and how do they perform? How can the resource (input), algae system and output be connected? How could we manage the construction process to realize such networks?

These questions call for a performance-based information system that integrates ArcGIS and BIM. In this system, ArcGIS and BIM generate both projectspecified data and urban-based data to improve the accuracy of planning models. At the same, their respective analytical tools enhance the overall performance analysis capacity of the model. The integration invites the extension of boundaries in the model and the thoroughness of the performance assessment. The data availability and analysis applicability in ArcGIS and BIM are shown in Table 2 ("G" means that the analysis can be done with GIS, while "B" means with BIM).

Table 2. Data and analysis in GIS and BIM

\begin{tabular}{|c|c|c|c|}
\hline Data & \multicolumn{3}{|c|}{ Analysis applicability } \\
\hline $\begin{array}{l}\text { BIM } \\
\text { data }\end{array}$ & $\begin{array}{l}\text { Solar } \\
\text { avail. }\end{array}$ & $\begin{array}{l}\text { Waste } \\
\text { stream }\end{array}$ & $\begin{array}{c}\text { Recycled } \\
\text { water }\end{array}$ \\
\hline location & $\mathrm{G}$ & & \\
\hline building system & & $\mathrm{B}$ & B \\
\hline \multirow{2}{*}{$\begin{array}{c}\text { urban infrastructure } \\
\text { massing }\end{array}$} & & G & G \\
\hline & & & \\
\hline \multirow{2}{*}{$\begin{array}{l}\text { building operation } \\
\text { fenestration }\end{array}$} & & $\mathrm{B}$ & $\mathrm{B}$ \\
\hline & B & & \\
\hline \multirow{3}{*}{ population } & & G & G \\
\hline & & B & B \\
\hline & Algal & Energy & Constr. \\
\hline
\end{tabular}

location cultiv. consum. mgmt.

dimension

\begin{tabular}{|c|c|c|c|}
\hline building system & B & & B \\
\hline urban infrastructure & G & & G \\
\hline massing & & BG & \\
\hline building operation & B & & \\
\hline fenestration & B & B & B \\
\hline population & & G & \\
\hline occupation & & B & \\
\hline material & & B & B \\
\hline
\end{tabular}

The integration of ArcGIS and BIM has different forms and applications at three scales. Figure 4 shows the management of the algal cultivation system in building construction using the integrated ArcGIS-BIM system.

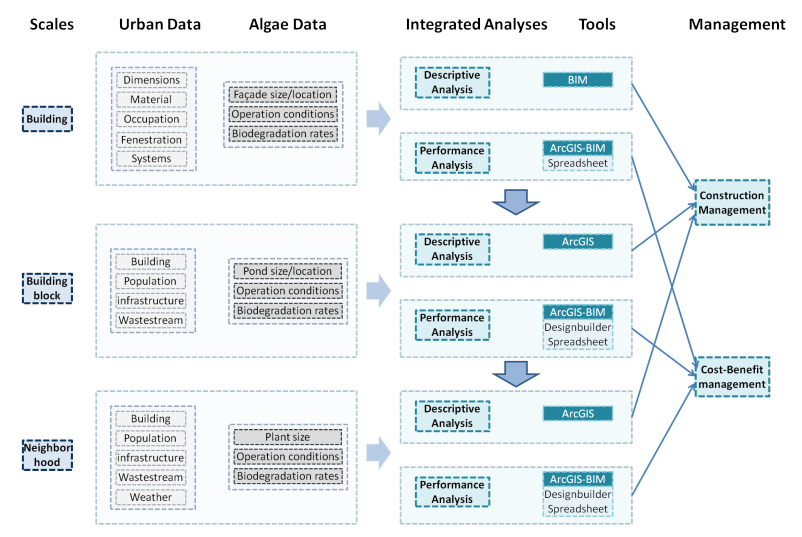

Figure 4. GIS-BIM informational system for managing algal cultivation system in residential construction

At the building scale, the BIM is intensively used to manage the data of material, fenestration, building-level algal system and to assess the waste stream, solar gain, energy consumption and input-output of the algal system. Then this information is transferred into ArcGIS as the node data in a network. At the building block scale, both ArcGIS and BIM are utilized. The dataset includes more information about the building block layout, the infrastructure network, the population and the algal pond operation data. The analysis extends from within the building to include the more accurate solar gain measurement with external shadings, as well as the network analysis with input and output of the algae system in buildings and in the ponds.

At the neighbourhood scale, the dataset and the analysis focus more on urban level. Using a GIS-based network analysis, the dynamic model can analyse both simple and complex waste stream and water recycle systems. GIS network analysis will provide opportunities for maximizing the distribution of waste and recycled water and minimizing construction costs. The analysis also supports all stages of waste stream 
management analysis, defining the topology of a sewer network, the specification of sewage flow contribution parameters, the allocation of sewage-contributing areas to sewer manholes, hydraulic analysis and displaying the analysis results, as illustrated in Figure 5.

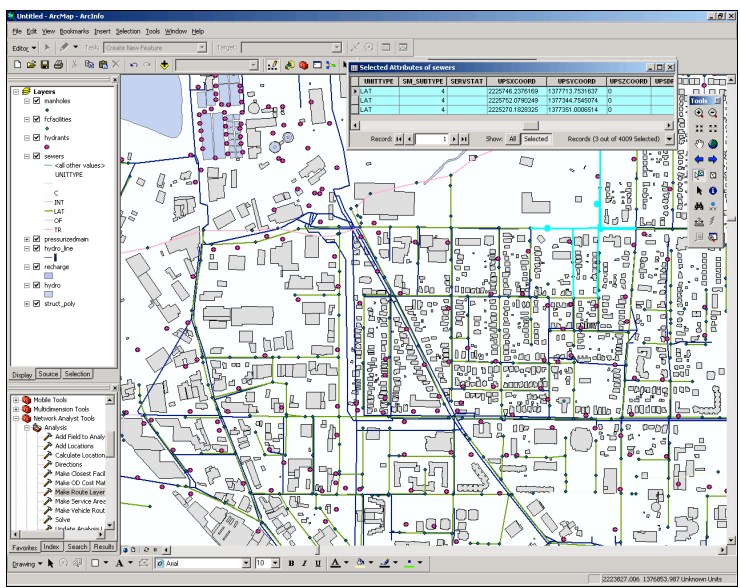

Figure 5. GIS-based network analysis

The solar radiation tool allows mapping and analysing the effects of the sun over a geographic area for specific time periods. It accounts for atmospheric effects, site latitude and elevation, steepness of slope and compass direction of aspect, daily and seasonal shifts of the sun angle, and effects of shadows cast by surrounding topography or buildings. The solar radiation tool calculates the sum of the direct radiation and diffuse radiation through sky. It is based on spherical approach of 3D GIS analysis developed by one of the authors [11]. The output radiation rasters will have units of watt hours per square meter $(\mathrm{WH} / \mathrm{m} 2)$.

\section{Integrating construction information}

The integrated framework incorporates construction information inherent to the facility, such as schedule, budgeted cost, and also has the possibility of including as-built cost, as well as constructability and safety considerations.

\subsection{Background and research questions}

There has been extensive research on recognizing construction elements on site and measuring progress using information technology [9, 12-15]. Construction progress monitoring is possible through a crosscorrelation of collected progress data using GIS and existing BIM information. This enhances the ability to determine progress by comparing the planned schedule and cost presented in the BIM model and actual progress, and helps field managers monitor the work progress conceptually. A robust system of performance metrics needs to back this process up. BIM comprises useful design information, overused during the design phase but overlooked during construction. Schedule and cost information provided by BIM and compared with GIS-based as-built data may provide decision makers with a useful tool for project monitoring with reliable progress indicators. Some research questions addressed by this approach include: what are the integration protocols required to compare BIM and GIS-based information from the construction of a single building, multifamily housing or a neighbourhood? Can Earned Value progress indicators be extracted from the comparison? How timely is the method to the decisionmakers?

A 5D (3D+time+cost) IFC-based model could be created in which as-built information is connected to BIM allowing a baseline for progress monitoring. The as-built cost information would be available on demand. This part of the research would analyse an automated Earned Value Analysis (EVA) system for progress monitoring based on comparing as-built with as-planned physical progress. Some of the research questions addressed include: how cost information could be linked up to $4 \mathrm{D}$ models to represent a rich baseline for asplanned data in Earned Value Analysis (EVA)? To what level of detail, 4D model needs to represent the asplanned progress? How non-constructing activities are represented in the as-planned model?

\subsection{Sources of preconstruction data}

A detailed cost estimate for the residential construction featuring the algae-based power system will follow Construction Specifications Institute (CSI) Masterformat, and will be properly populated with cost data from standard manuals (e.g., RS Means, Timberline), local builders, specialist trade subcontractors, and material suppliers. Costs will be adjusted based on geographic location, and the standard manuals will account for the adjustment. Also, local contractors and material suppliers can provide relevant prices of construction materials, equipment, and labour. Scheduling information will be computed from the standards manuals using daily production values for labour and material installation, and expected durations from equipment productivity manuals. Specific tasks to be included in the estimate include: 
1. Investigate the costs of installing and putting in operation the algae system, including the collection of wastewater (e.g., toilets, sinks, showers); delivery to the pond; transfer to the bioreactor; installation of bioreactor and all ancillary systems

2. Establish a maintenance plan for all systems mentioned above and estimate training requirements for safe and on-going operation.

3. Calculate life expectancy of the system, as a whole or its parts and estimate the replacement cost.

4. Analyse entire value chain from a cost perspective; track and evaluate costing from the research and development phase of the system's life, through to the decline and eventual conclusion of its life.

Additional considerations that impact cost are associated with the life cycle assessment, ownership, delivery, and technology implementation. Such considerations include:

1. Evaluate the system's environmental aspects and its potential impacts in general and specifically. Assess the raw materials (inputs) needed to effectively operate the system and its probable releases to the environment.

2. Investigate possible reuse, maintenance and waste management.

3. Investigate the embodied energy of the system put in place and operating.

4. Analyse different deployment schemes for the system: plug-and-play, when the decision to invest in the system is made by the owner or occupier of the dwelling; company control: a group of consumers provide the site for the system to be installed and it is operated by an Energy Service Company (ESCo) or other third-party energy supplier; community micro grid: a group of individuals groups together to provide some or all of their collective energy needs. They own and operate the system.

5. Investigate different forms of ownership for end users and their implications in initial and running costs, maintenance and upgrades.

6. Establish training levels and parameters for the different deployment schemes.

7. Study the delivery of the service and rate structures.
8. Most systems being evaluated are not mainstream, from water separating toilets, sinks, etc., to the retention ponds for wastewater and the bioreactor itself. Investigate the safety implications of installing such a system in a residential environment. For example: maintaining a wastewater pond in proximity to residential dwellings is not the same as a storm water retention pond. Determine the real and perceived safety issues related to the installation and operation of such a system of energy generation.

9. Determine the different levels of control needed to operate the system safely and who is better qualified to handle the risk.

10. Management: what are the personnel, cost and liability implications of maintaining safety while operating the system.

11. Design (differences with existing systems affected): study the design and construction implications and code issues related to implementing the design in a typical residential environment

12.Experiment and model issues of scalability. Siting concerns, size of reactor, size of wastewater pond, distribution of energy produced, maintenance, handling of by-products and recycling.

\subsection{Comparing preconstruction and as-built data}

As-built elements will be generated from GIS for outdoor or visible objects as they are being installed. This information will be moved to the data fusion bin, or an IFC-compatible file that features $[\mathrm{x}, \mathrm{y}, \mathrm{z}]$ coordinates in raster version, as well as element properties and sequencing information in $3 \mathrm{D}$ Object Exchange format. The coordinate origin $[0,0,0]$ is elicited from the BIM model, and the $\mathrm{x}, \mathrm{y}$, and $\mathrm{z}$ positions of each tag in feet or meters are defined with respect to that origin, thereby constructing an IFC file. This file will be merged to the existing GIS data in the data fusion bin. In the third step, a task allocation protocol activated in the data fusion bin will revise the integrity of the data as to contain $[\mathrm{x}, \mathrm{y}, \mathrm{z}]$ coordinates of the objects' centroids and exterior corners. Data, formatting integrity and file compatibility will be periodically checked for comparison adequacy with IFC-based as-planned data provided by BIM, as well as to generate progress reports and visualization protocols. A client program using stream communication protocol will be required to retrieve data from the IEEE 802.11 network hub and to establish the parameters by which the data fusion bin will proceed with the combination with GIS-based data, as specified in the task allocation 
protocol. The default data output stream provided from the processing hub to the client program is:

$<$ Data Header $>, \quad<\operatorname{tag} \#>, \quad<\mathrm{X}>, \quad\langle\mathrm{Y}\rangle, \quad<\mathrm{Z}>$, $<$ timestamp $>$, $<$ unit $><$ LF $>$

The data output from the hub can be modified to provide increased resolution in the timestamp field. $<$ Data Header $>$ represents the tag dimensional information. Expected values for $<$ Data Header $>$ are nomenclature representing construction elements. For example, $\mathrm{C} \#$, which indicates an internal column with an extension number whose value will denote possible configurations as specified in the BIM model; $<\operatorname{tag} \#>$ is the tag ID; $<\mathrm{X}>,<\mathrm{Y}>,\langle\mathrm{Z}>$, are the calculated tag coordinates in feet or meters with respect to a user supplied origin; <timestamp $>$ represents the hub system time. The format for timestamp is UTC time, day, and year the data was computed. An additional data field is also programmed to indicate the quality of data provided from the hub and the type of element being positioned; finally, $<\mathrm{LF}>$ is a Line Feed character (with ASCII code $=0 \times 0 \mathrm{~A}$ ), to terminate a location data string. This data structure will enter the data fusion bin, where it will be merged with IFC-based GIS data through a task allocation protocol.

In order to perform the element matching, a software application will be developed to export the data fusion bin, using an IFC Industry Foundation Classes format (based on the ISO-STEP EXPRESS language which is an industry standard for building information exchange and consistent data representations), to a BIM application (i.e., Revit, ArchiCAD, Bentley). The data file will have the following structure:

ISO-11253-32;

HEADER

FILE_DESCRIPTION (\$):

FILE NAME (\$,'Sat Jan 25 14:03:45

2014',(Ā C $),($ 'Shingle'),'ASX_Roof

v.0.1'ASX_Project',\$);

FILE SCHEMA ('ASX');

ENDSEC;

DATA;

$\# 1=$ CATALOG ('Argos1 (644)', 'Argos1

CONSTRUCTION LIBRARY');

\#2 = GEOMETRY ('Argos1 (101)’, 0.58, \$,'Corner', 'Real');

\#3 = BOUNDARY ('Argos1 (102)', 0.61, \$,

'Boundaries', 'Real');

$\# 4=$ SCHEDULE ('Argos1 (103)’, 0.63, \$,

'Timestamp', Real');

ENDSEC;

END-ISO-11253-32;

The BIM application generates the as-built model with the data imported, superposing both GIS and BIM models.

\section{Conclusions}

This paper addresses a conceptual model for integrating input/output information involved in developing an innovative technology for providing reliable and efficient power generation to improve the energy consumption in American homes. This model impacts the design of household and residential communities: by integrating domestic waste stream management with the concurrent production of energy, substantial reductions in energy and significant conservation of water and waste can be achieved. Algae technology has emerged as a promising option due to its closed-loop configuration and the ability to produce biofuel energy while reducing waste stream flow and capturing carbon. This paper provides a framework for integrating information from geographic information systems, building information models, construction schedules, construction cost estimates, and constructability reviews. The integration is aimed at designing an algae-powered residential building environment at the level of urban neighbourhood, in which the algae technology is taken as a design intervention to promote energy performance and carbon reduction within the urban system. This framework couples the design intervention with impact simulations influenced by geographic contexts, construction considerations, and digital building technology. By extending the system boundary from a closed algae cultivation system to an open neighbourhood-scale urban environment, urban renewable resources such as energy, water, material and carbon flows are connected to the algae cultivation process. The framework would further advance the possibilities for sharing information among planners, architects, engineers and construction managers for innovative closed-loop sustainable energy systems in residential construction. This approach will address challenges such as cost, governmental incentives, regulatory barriers, or need of research and development that could overcome limitations for automating predesign, design, construction and facility management

\section{References}

[1] Scott S.A., Davey M.P., Dennis J.S., Horst I., Howe C.H., Lea-Smith D.J., and Smith D.G. Biodiesel from algae: challenges and prospects, Current Opinion in Biotechnology, 21(3): 277-286, 2010.

[2] Amaroa H.M., Guedesb A.C., and Malcatac F.X. Advances and perspectives in using microalgae to produce biodiesel. Applied Energy, Special Issue of Energy from Algae: Current Status and Future Trends, 88(10): 3402-3410, 2011. 
[3] Demirbas M.F. Biofuels from algae for sustainable development. Applied Energy, Special Issue of Energy from Algae: Current Status and Future Trends, 88(10): 3473-3480, 2011.

[4] NASA. NASA Envisions clean energy from algae grown in waste water. Online: http://www.nasa.gov/centers/ames/news/features/20 09/clean energy 042209.html, Accessed 22/01/2014.

[5] Randolph, J., Environmental Land Use Planning. Island Press, 2003.

[6] United States Environmental Protection Agency. Low impact development. Online: http://www.epa.gov/nps/lid/, Accessed 22/01/14.

[7] Pauleit, S. and Duhme, F. Assessing the environmental performance of land cover types for urban planning, Landscape and Urban Planning, 52: 1-20, 2000.

[8] Scheer, D.R. and R.E. Smith, Download my building: How building information modeling will transform our cities, in Societies and Cities in the Age of Instant Access, H.J. Miller, Editor. 2007, Springer: Netherlands.

[9] Eastman, C., Teicholz, P., Sacks, R., and Liston, K. BIM Handbook: A guide to building information modeling for owners, managers, designers, engineers, and contractors, John Wiley \& Sons, New Jersey, 2008.

[10] Goodchild, M.F., Twenty years of progress: GIScience in 2010. Journal of Spatial Information Science, 2010(1).

[11] Yang, P.P.J., Putra, S.Y. and Li, W. Viewsphere: GIS-based 3D visibility analysis for urban design evaluation. Environment and Planning B: Planning and Design, 34(6): 971-992, 2007.

[12] Navon, R. Research in automated measurement of project performance indicators, Automation in Construction, 16(7): 176-188, 2006.

[13] Akinci, B., Patton, M. and Ergen, E. Utilizing radio frequency identification on precast concrete components- supplier's perspective, Proc. of ISARC- 19th Int. Symp. on Automation and Robotics in Construction, National Institute of Standards and Testing, pages 381-386, 2002.

[14]Peyret, F., Betaille D., and Hintzy G. Highprecision Application of GPS in the field of realtime equipment positioning, Automation in Construction, 9 (3): 299-314. 2000.

[15] Castro-Lacouture, D., Bryson, L.S., and GonzalezJoaqui, J. Real-time positioning network for intelligent construction, Proceedings of the Joint International Conference on Computing and
Decision Making in Civil and Building Engineering, Montreal, Canada, 77-86, 2006. 